\title{
Propriedades de um Argissolo Amarelo fragipânico de Alagoas sob cultivo contínuo da cana-de-açúcar
}

\author{
Joselanne Luiza Trajano Maia(1) e Mateus Rosas Ribeiro(2)
}

(1)Embrapa Semi-Árido, Caixa Postal 23, CEP 56302-970 Petrolina, PE. Bolsista do CNPq. E-mail: luizatm@cpatsa.embrapa.br (2)UFRPE, Dep. de Agronomia, R. Dom Manoel de Medeiros, s/nº, Dois Irmãos, CEP 52171-900 Recife, PE. Bolsista do CNPq. E-mail: rosas@truenet.com.br

\begin{abstract}
Resumo - O objetivo deste trabalho foi avaliar as alterações nas propriedades morfológicas e físicas de um Argissolo Amarelo distrófico abrupto fragipânico sob cultivo contínuo de cana-de-açúcar. Três talhões foram selecionados, um coberto por vegetação de mata nativa e os outros dois cultivados com cana-de-açúcar, por períodos de dois e trinta anos. O cultivo da cana-de-açúcar alterou morfologicamente o horizonte superficial, desenvolvendo um horizonte Ap, e modificou a estrutura dos dois primeiros horizontes do perfil. As alterações morfológicas, entretanto, foram pouco expressivas em virtude dos baixos teores de argila destes horizontes. O manejo adotado causou diminuição da macroporosidade e, consequentemente, aumentou a água disponível. Por outro lado, o uso agrícola aumentou a microporosidade e reduziu significativamente a condutividade hidráulica dos horizontes superficiais. Não se observou variação significativa na densidade do solo, porosidade total, macroporosidade, ponto de murcha permanente ou no tamanho e distribuição dos agregados. Constatou-se desenvolvimento de compactação nos horizontes $\mathrm{Ap}$ e $\mathrm{AB}$, em decorrência do cultivo da cana-de-açúcar, e a existência de adensamento (caráter coeso) no horizonte $\mathrm{Bt}$ de todos os perfis.
\end{abstract}

Termos para indexação: Saccharum, morfologia de solo, compactação do solo, propriedade física do solo.

\section{Properties of a Fragiudult in Alagoas State, Brazil, under continuous sugarcane cultivation}

\begin{abstract}
The objective of this work was to evaluate the modifications on morphological and physical properties of a Fragiudult caused by continuous sugarcane cultivation in the Low Coastal Tablelands region of Alagoas State, Brazil. Three sites were selected, one from native forest and two cultivated with sugarcane for periods of two and thirty years. The sugarcane cultivation promoted morphological changes on the soil surface with the development of an Ap horizon, and changed the soil structure of the two upper horizons. The morphological changes, however, were not expressive due to the small clay content of the horizons. The cropping practices adopted decreased macroporosity and, consequently, increased the soil water availability. Land use increased microporosity and decreased significantly the hydraulic conductivity of the surface horizons. Significant changes in bulk density, total porosity, macroporosity, wilting point, and in the size and distribution of aggregates were not observed. The development of compaction on the Ap and AB horizons as a result of sugarcane cultivation, and the presence of a dense layer (cohesion) in the Bt horizon of all soil profiles were observed.
\end{abstract}

Index terms: Saccharum, soil morphology, soil compaction, soil physical properties.

\section{Introdução}

No Brasil, a lavoura da cana-de-açúcar ocupa cerca de 4,4 milhões de hectares, que correspondem a $30 \%$ da área canavieira mundial. Destes, aproximadamente $70 \%$ são destinados à produção de álcool e $30 \%$ à de açúcar (Urquiaga et al., 1996). No Nordeste, o Estado de Alagoas tem uma produtividade de cana-de-açúcar superior à dos demais, destacando-se como o maior produtor de açúcar e álcool da região (Lima, 1997).
Com a retirada da vegetação natural e subseqüentes anos de cultivo, ocorre uma diminuição gradativa da aptidão cultural do solo, pela alteração de suas propriedades físicas (Cavenage et al., 1999). A intensidade da degradação nas propriedades físicas do solo e as alterações nos fatores físicos de crescimento variam com os diferentes sistemas de manejo e preparo adotados e com o tempo de uso e, na maioria dos casos, está associada ao manejo intensivo do solo (Cintra, 1980). 
O cultivo contínuo, o preparo do solo para o plantio pela gradagem e o uso freqüente de cultivadores, na maioria das vezes, não são realizados nas condições ideais de umidade. Essas ações provocam diversas alterações nas propriedades físicas, resultando em uma degradação da estrutura do solo, principalmente em relação à distribuição do tamanho de agregados estáveis em água (Da Ros et al., 1997).

Os solos podem estar compactados naturalmente ou atingirem esse estado mediante práticas de manejo impostas pelo cultivo. A compactação e o adensamento da camada arável podem afetar drasticamente o desenvolvimento radicular e os processos de armazenagem e disponibilidade de água, podendo acarretar redução dos efeitos da adubação mineral e da irrigação, diminuindo o rendimento das culturas, apesar de toda tecnologia empregada (Camargo, 1983). A presença de camadas adensadas de origem pedogenética ou antrópica, refletindo no crescimento e na produção da planta, foi também citada por Paiva (1997).

O estudo do efeito de diferentes métodos de cultivo em um Latossolo Amarelo argiloso revelou que os sistemas de preparo da área alteraram, com diferentes intensidades, a densidade do solo na camada de $0-10 \mathrm{~cm}$, com valores significativamente superiores em relação à floresta nativa (Correia, 1985). Resultados semelhantes foram verificados por Mahboubi et al. (1993).

Ao longo do tempo, o cultivo contínuo do solo diminui a porosidade total, aumenta a densidade do solo e reduz a taxa de infiltração e, conseqüentemente, diminui também a rentabilidade, em decorrência do crescente aumento dos custos de produção (Silva, 1980). A condutividade hidráulica do solo diminui, com o aumento da sua densidade, assim como a taxa de infiltração em solos compactados, causando aeração deficiente e aumento do escoamento superficial de água. Tal comportamento é capaz de acelerar o carreamento de partículas do solo juntamente com fertilizantes e defensivos agrícolas (Camargo \& Alleoni, 1997).

O comportamento do solo quanto à porosidade é um reflexo de diversos fatores inter-relacionados, como por exemplo, a ação de máquinas agrícolas, que exerce pressão sobre o solo, causando alterações nas suas propriedades físicas, como a redução da porosidade total (Wu et al., 1995).

No Estado de Alagoas, a maior parte das áreas cultivadas com cana-de-açúcar está localizada em solos de Tabuleiros Costeiros, que se caracterizam pela baixa fertilidade. Com o avanço tecnológico, os problemas de fertilidade foram superados pelo desenvolvimento de práticas aparentemente adequadas, mas que podem, a longo prazo, provocar danos às propriedades físicas desses solos, que mostram forte coesão natural em subsuperfície (Silva \& Ribeiro,1997).

A falta de dados precisos sobre as alterações físicas dos solos sob cultivo contínuo de cana-de-açúcar, nestas áreas, tem dificultado a identificação de fatores responsáveis pelo decréscimo da produtividade, bem como a implantação de práticas mais adequadas à manutenção das condições agrícolas.

O objetivo deste trabalho foi avaliar alterações nas propriedades morfológicas e físicas de um Argissolo Amarelo distrófico abrupto fragipânico, sob cultivo contínuo de cana-de-açúcar.

\section{Material e Métodos}

O trabalho foi realizado nas áreas da Usina Coruripe, no Município de Coruripe, zona fisiográfica do Litoral do Estado de Alagoas (Embrapa, 1975). Foram selecionados três perfis de Argissolo Amarelo distrófico abrupto fragipânico, A moderado, textura média (leve)/argilosa, representativos de áreas com vegetação nativa e cultivadas com cana-de-açúcar por diferentes períodos de tempo, com base em um levantamento pedológico detalhado (Usina Coruripe, 1997).

Os solos com diferentes períodos de cultivo foram comparados entre si e em relação a uma testemunha absoluta, representada pelo solo em condições naturais (vegetação nativa), recebendo as seguintes identificações: Tn solo sob vegetação de mata nativa; T2 solo com dois anos de cultivo com cana-de-açúcar; T30 solo com trinta anos de cultivo com cana-de-açúcar. Os perfis localizaram-se em uma mesma situação topográfica de topo plano, de baixo platô costeiro (tabuleiro).

$O$ perfil Tn se caracteriza por uma sequiência de horizontes $\mathrm{Oo}, \mathrm{A}, \mathrm{AB}, \mathrm{Bt}$ e $\mathrm{Btx}$, com transição clara do $\mathrm{A}$ para o $\mathrm{AB}$ e abrupta entre os demais horizontes. Apresenta uma fina camada orgânica (Oo), formada por restos vegetais pouco decompostos, seguida por um horizonte A, de coloração bruno-acinzentada muito escura (10YR 3/2), quando úmido, textura areia franca e estrutura fraca, pequena a média granular, com consistência ligeiramente dura e friável. $\mathrm{O}$ horizonte $\mathrm{AB}$ apresenta coloração bruna (10YR 5/3), quando úmido, textura franco arenosa, estrutura fraca, pequena a média granular, e consistência friável, quando úmido. 
O horizonte Bt tem cor bruno-amarelada-clara (10YR 6/4), com mosqueado distinto bruno-forte (7,5YR 5/6), textura argilo-arenosa, estrutura maciça coesa, e consistência extremamente dura e firme, preenchendo os requisitos de um horizonte coeso, que tem origem pedogenética, conforme Silva \& Ribeiro (1992) e Silva, \& Ribeiro (1997). O horizonte Btx tem coloração bruno-amarelada-clara (10YR 6/4), com mosqueados abundantes, médios e grandes; nas cores vermelho $(2,5 \mathrm{YR}$ 4/6), vermelho-amarelado (5YR 5/6) e bruno-amarelado (10YR 5/6). O Btx tem textura argilosa, estrutura maciça coesa e laminar, e consistência extremamente dura e muito firme. A caracterização morfológica dos perfis foi feita segundo Lemos \& Santos (1996).

O manejo adotado nas áreas cultivadas envolve, no primeiro plantio e a cada seis anos, quando é feita a renovação do canavial, correção da acidez, gradagem e subsolagem, seguida do plantio de leguminosa, que é, posteriormente, incorporada por meio de gradagem, sendo então feito o sulcamento, adubação com N P K, de acordo com a análise do solo, e o plantio. Anualmente, após a colheita da cana-de-açúcar queimada, é feita uma subsolagem rasa, com aplicação de matéria orgânica (composto) e adubação. Nos últimos dois anos, a área T30 recebeu $350 \mathrm{~m}^{3} \mathrm{ha}^{-1}$ de vinhaça, em duas aplicações por ano.

As amostras foram coletadas em todos os horizontes, após a descrição morfológica dos perfis, e secadas ao ar, destorroadas e passadas em peneiras de $2 \mathrm{~mm}$ para obtenção da TFSA (terra fina secada ao ar). Foram também coletadas amostras não deformadas para determinação da densidade do solo, microporosidade, condutividade hidráulica saturada e diâmetro médio dos agregados.

As análises físicas foram realizadas no laboratório de Física do Solo da Universidade Federal Rural de Pernambuco, segundo métodos descritos no Manual de Métodos de Análises de Solo (Embrapa, 1997). Os parâmetros físicos determinados foram: densidade do solo com anel volumétrico; densidade das partículas com balão volumétrico; microporosidade com amostras não deformadas, utilizando o funil de Buchner; porosidade total, segundo Vomocil (1965); macroporosidade, conforme Grohmann (1960); granulometria pelo método do densímetro de Boyoucos; argila dispersa em água pelo mesmo método da granulometria; capacidade de campo e ponto de murcha permanente (PMP), segundo Richards (1969); água disponível; condutividade hidráulica saturada, em amostras não deformadas, por meio de permeâmetro, descrito pela Embrapa (1979) e conforme a equação de Darcy-Williams, descrita por Klute \& Dirksen (1986); disponibilidade total de água, calculada por meio da expressão: DT $=(C C$ - PMP $) \times D g / 10$; e estabilidade dos agregados, em que o solo foi coletado não deformado, nas condições de campo, secado ao ar, passado em peneira com $4 \mathrm{~mm}$ de malha, colocando $100 \mathrm{~g}$ de cada amostra na peneira superior, em um jogo de peneiras de 2, 1, 0,5 e 0,25 mm, e agitado em aparelho de oscilação vertical, perfazendo um total de 30 imersões por amostra, segundo método descrito pela Embrapa (1979). Determinou-se ainda o carbono orgânico, pelo método volumétrico com oxidação pelo dicromato de potássio.

As variações das propriedades do solo com o tempo de cultivo e com a profundidade foram estudadas por meio do esquema fatorial $3 \times 3$, correspondente a uma parcela de referência ( $\mathrm{Tn})$ e dois diferentes tempos de cultivo (T2 e T30), e três horizontes, e analisadas por meio um delineamento inteiramente casualizado (Silva \& Silva, 1995). Além das amostras coletadas nos horizontes de cada perfil, foram coletadas mais quatro amostras dos três primeiros horizontes $(\mathrm{A}, \mathrm{AB} \mathrm{e} \mathrm{Bt})$, por meio de tradagens, em pontos diferentes do talhão, perfazendo cinco repetições. Quando o efeito do tempo de cultivo ou da profundidade, bem como das interações entre eles, foram significativos pelo teste $\mathrm{F}$, a comparação das médias foi feita pelo teste de Tukey a 5\% de probabilidade, usando-se o software SAS/STAT (SAS Institute, 1999).

\section{Resultados e Discussão}

Todos os perfis foram classificados como Argissolo Amarelo distrófico abrupto fragipânico, A moderado, textura média (leve)/argilosa, fase floresta subperenefólia, relevo plano. A seqüência de horizontes nos perfis estudados foi A ou Ap, AB, Bt e Btx. O perfil T30 apresentou uma subdivisão do $\mathrm{Bt}$, em $\mathrm{Bt}_{1}$ e $\mathrm{Bt}_{2}$, em conseqüência da posição mais profunda do horizonte subjacente (Btx), sem nenhuma relação com a utilização agrícola.

O perfil Tn apresentou um horizonte orgânico (Oo) de $0,04 \mathrm{~m}$ de espessura, formado por restos vegetais pouco decompostos. Nos perfis cultivados, este horizonte orgânico foi queimado ou incorporado pelo cultivo ao horizonte $\mathrm{A}$, formando o Ap. O horizonte superficial não apresentou variação expressiva de profundidade entre os perfis. Já o horizonte $A B$ apresentou espessura entre 0,12 e $0,18 \mathrm{~m}$, com o menor valor correspondendo 
ao solo com maior tempo de cultivo. A espessura do Bt variou entre 0,38 e $0,61 \mathrm{~m}$, principalmente em conseqüência das diferentes profundidades de ocorrência do Btx, que teve início a cerca de $0,75 \mathrm{~m}$ de profundidade nos perfis T2 e Tn, e aos 0,90 m no T30 (Tabela 1). Esta variação na profundidade de ocorrência do fragipã é uma característica comum nos tabuleiros costeiros do terciário. Quanto à cor e textura, os horizontes superficiais e subsuperficiais são muito uniformes, quando comparados entre si e para os três perfis de solo.

Em todos os perfis, as transições entre os horizontes são claras, do A ou Ap para o AB e abruptas para o horizonte Bt. A utilização agrícola provocou variações apenas na topografia das transições dos horizontes superficiais. Do horizonte A para o AB, passou de plana no perfil Tn, para ondulada nos perfis T2 e T30, por causa das operações de sulcamento no plantio. No perfil T30 a topografia da transição de AB para Bt foi modificada de plana para ondulada, em consequiência da prática da subsolagem profunda, que é feita quando da renovação do canavial, de seis em seis anos.

Apesar do baixo teor de argila dos horizontes superficiais, foram observadas pequenas modificações da estrutura com o tempo de cultivo, tanto no grau de desenvolvimento como no tipo. No horizonte superficial A ou Ap, observou-se que a estrutura passou de fraca, pequena a média, granular, no perfil Tn, para uma combinação de estrutura maciça, pouco coesa e fraca, pequena a média, granular nos demais perfis. A estrutura do horizonte $\mathrm{AB}$ também sofreu modificações com o cultivo, passando de fraca, pequena a média, granular, no perfil Tn, para maciça moderadamente coesa e fraca, pequena a média, blocos subangulares, no T2, e maciça moderadamente coesa, ou seja, sem nenhuma agregação, no perfil T30. Esta alteração de estrutura ocorreu em consequiência da compactação provocada pelo cultivo, promovendo uma destruição parcial dos agregados no perfil T2 e total no T30. Os horizontes subsuperficiais Bt e Btx não sofreram influência do cultivo. A estrutura do Bt é maciça coesa em todos os perfis, em conseqüência da coesão pedogenética encontrada em algumas classes de solos de tabuleiro, como também observado por Silva \& Ribeiro (1992), em Podzólicos Amarelos latossólicos, e por Silva \& Ribeiro (1997) em Latossolos Amarelos coesos. No horizonte Btx, a estrutura variou com o grau de desenvolvimento do horizonte fragipã, sendo maciça coesa e laminar no perfil Tn, maciça coesa no T30 e fraca, pequena a média, e em blocos angulares no T2.
Nos horizontes A e AB de todos os perfis, a consistência do material úmido foi friável. No solo seco, a consistência foi ligeiramente dura no A, não sendo feita essa determinação no AB. Os horizontes subsuperficiais Bt e Btx apresentaram, em todos os perfis, consistência extremamente dura e firme. No Bt, esta consistência é decorrente do seu caráter coeso, e tem origem pedogenética, como sugerido por Silva \& Ribeiro (1992) e Silva \& Ribeiro (1997). No Btx a consistência extremamente dura é determinada pelo caráter fragipã, que reflete as condições de drenagem dos perfis. No solo molhado, as consistências foram muito semelhantes e dependentes, exclusivamente, dos teores de argila, visto que os solos têm a mesma mineralogia. Apresentou-se não plástica e não pegajosa no A, ligeiramente plástica e ligeiramente pegajosa no $\mathrm{AB}$, e plástica ou muito plástica, e pegajosa ou muito pegajosa em Bt e Btx. Nos perfis Tn e T30, observou-se uma diminuição da plasticidade e, principalmente, da pegajosidade no Btx, em relação ao horizonte $\mathrm{Bt}$, independentemente da variação no teor de argila.

Não houve variações significativas nos teores de areia, silte e argila dos horizontes $\mathrm{A}, \mathrm{AB}$ e $\mathrm{Bt}$, com o tempo de cultivo, exceto com relação aos teores de silte, que apresentaram aumento significativo no perfil T30 (Tabela 2).

Com relação à profundidade, houve um aumento significativo do teor de argila e uma redução no teor de areia e silte em todos os perfis, o que é esperado, tendo em vista a classe de solo identificada (Argissolo). Os teores de argila aumentaram com a profundidade, e do horizonte $\mathrm{AB}$ para o $\mathrm{Bt}$ aumentaram bruscamente, promovendo uma mudança textural abrupta. No Btx os teores de argila aumentaram nos perfis T2 e T30 e apresentaram uma redução no perfil Tn, provavelmente, em conseqüência do maior grau de desenvolvimento (cimentação) do horizonte fragipã neste perfil, avaliado pela presença de estrutura laminar, aumento do mosqueado vermelho e consistência muito firme.

A Tabela 3 mostra um aumento nos valores da densidade do solo dos horizontes Ap e AB dos perfis cultivados, em relação aos do Tn. No horizonte Ap, o aumento da densidade do solo foi mais expressivo no perfil T2, demonstrando que a remoção da vegetação nativa e a implantação da cultura da cana-de-açúcar promoveram a destruição da estrutura e da matéria orgânica. No perfil T30, o valor da densidade do solo decresceu em relação ao T2, provavelmente por causa do cultivo, da incorporação da matéria orgânica e do sistema radicular fasciculado da cana-de-açúcar, resultados também ob- 


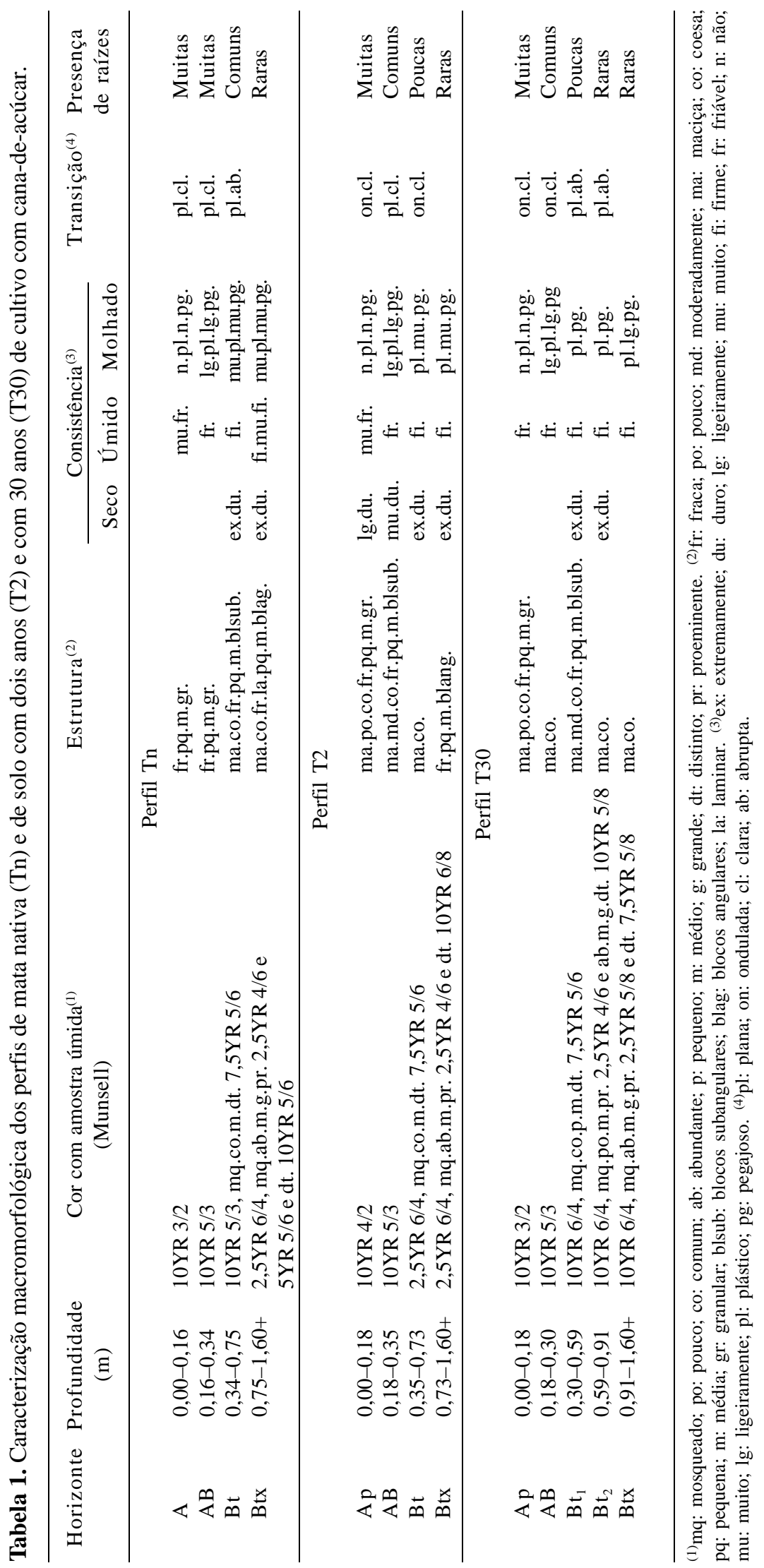

Pesq. agropec. bras., Brasília, v.39, n.1, p.79-87, jan. 2004 
servados por Silva (1996), em Latossolos Amarelos. No horizonte $\mathrm{AB}$, os maiores valores da densidade do solo foram observados no perfil T30. Isto demonstra que quanto maior o tempo de cultivo, maior a compactação resultante do tráfego de máquinas e implementos, considerando que este horizonte não é afetado pelas operações de gradagem. Com relação ao Bt, o maior valor da densidade do solo foi verificado no perfil Tn, demonstrando que se trata de um adensamento pedogenético, característica comum aos solos dos tabuleiros costeiros, corroborando os resultados observados por Silva \& Ribeiro (1992) em Podzólicos Amarelos latossólicos e por Silva \& Ribeiro (1997) em Latossolos Amarelos coesos, ambos no Estado de Alagoas.

Tabela 2. Granulometria e classificação textural e carbono orgânico dos perfis de mata nativa (Tn) e de solo com dois anos (T2) e com 30 anos (T30) de cultivo com cana-de-açúcar, com relação textural de 2,93, 3,15 e de 3,00, respectivamente, em razão do tempo de cultivo e dos horizontes ${ }^{(1)}$.

\begin{tabular}{|c|c|c|c|c|c|}
\hline Horizonte & Areia & $\begin{array}{r}\text { Silte } \\
\left(\mathrm{g} \mathrm{kg}^{-1}\right)\end{array}$ & Argila & Classificação textural & $\begin{array}{c}\text { Carbono orgânico } \\
\qquad\left(\mathrm{g} \mathrm{kg}^{-1}\right)\end{array}$ \\
\hline \multicolumn{6}{|c|}{ Perfil Tn } \\
\hline A & 830Aa & $60 \mathrm{Ba}$ & $110 \mathrm{Ac}$ & Areia franca & $2,12 \mathrm{Aa}$ \\
\hline $\mathrm{AB}$ & $790 \mathrm{Ab}$ & $40 \mathrm{Bb}$ & $170 \mathrm{Ab}$ & Franco arenoso & $1,02 \mathrm{Ab}$ \\
\hline $\mathrm{Bt}$ & $560 \mathrm{Ac}$ & $30 \mathrm{Bb}$ & 410Aa & Argila arenosa & $0,74 \mathrm{Ab}$ \\
\hline Btx & 540 & 90 & 370 & Argila arenosa & \\
\hline \multicolumn{6}{|c|}{ Perfil T2 } \\
\hline Ap & $850 \mathrm{Aa}$ & $50 \mathrm{Ba}$ & $100 \mathrm{Ac}$ & Areia franca & $1,09 \mathrm{Ba}$ \\
\hline $\mathrm{AB}$ & $810 \mathrm{Ab}$ & $30 \mathrm{Bb}$ & $160 \mathrm{Ab}$ & Franco arenoso & $0,71 \mathrm{Bb}$ \\
\hline $\mathrm{Bt}$ & $560 \mathrm{Ac}$ & $30 \mathrm{Bb}$ & 410Aa & Argila arenosa & $0,52 \mathrm{Bb}$ \\
\hline Btx & 380 & 100 & 520 & Argila & \\
\hline \multicolumn{6}{|c|}{ Perfil T30 } \\
\hline Ap & 800Aa & 70Aa & $130 \mathrm{Ac}$ & Franco arenoso & $1,22 \mathrm{ABa}$ \\
\hline $\mathrm{AB}$ & $770 \mathrm{Ab}$ & $50 \mathrm{Ab}$ & $180 \mathrm{Ab}$ & Franco arenoso & $0,92 \mathrm{ABb}$ \\
\hline $\mathrm{Bt}_{1}$ & $530 \mathrm{Ac}$ & $50 \mathrm{Ab}$ & $420 \mathrm{Aa}$ & Argila arenosa & $1,00 \mathrm{ABb}$ \\
\hline $\mathrm{Bt}_{2}$ & 400 & 90 & 510 & Argila & \\
\hline Btx & 340 & 160 & 500 & Argila & \\
\hline
\end{tabular}

${ }^{(1)}$ Médias seguidas das mesmas letras, maiúsculas nas linhas e minúsculas nas colunas, não diferem entre si a 5\% de probabilidade pelo teste de Tukey.

Tabela 3. Densidade do solo, microporosidade, macroporosidade e porosidade total, em razão do tempo de cultivo e dos horizontes dos perfis de mata nativa (Tn) e de solo com dois anos (T2) e com 30 anos (T30) de cultivo com cana-de-açúcar(1).

\begin{tabular}{|c|c|c|c|c|c|}
\hline \multicolumn{2}{|c|}{ Horizonte } & \multirow{2}{*}{$\begin{array}{l}\text { Densidade do solo } \\
\qquad\left(\mathrm{mg} \mathrm{m}^{-3}\right)\end{array}$} & \multicolumn{3}{|c|}{ Porosidade (\%) } \\
\hline Símbolo & Expessura (m) & & Micro & Macro & Total \\
\hline \multicolumn{6}{|c|}{ Perfil Tn } \\
\hline A & $0,00-0,16$ & 1,19Aa & $17 \mathrm{Ba}$ & $38 \mathrm{Aa}$ & $55 \mathrm{Aa}$ \\
\hline $\mathrm{AB}$ & $0,16-0,34$ & $1,41 \mathrm{Aa}$ & $20 \mathrm{Ba}$ & $27 \mathrm{Aa}$ & 47Aa \\
\hline $\mathrm{Bt}$ & $0,34-0,75$ & $1,57 \mathrm{Aa}$ & $19 \mathrm{Ba}$ & $24 \mathrm{Aa}$ & $43 \mathrm{Aa}$ \\
\hline \multicolumn{6}{|c|}{ Perfil T2 } \\
\hline Ap & $0,00-0,18$ & $1,51 \mathrm{Aa}$ & $26 \mathrm{ABa}$ & $17 \mathrm{Aa}$ & $43 \mathrm{Aa}$ \\
\hline $\mathrm{AB}$ & $0,18-0,35$ & $1,59 \mathrm{Aa}$ & $21 \mathrm{ABa}$ & $17 \mathrm{Aa}$ & $40 \mathrm{Aa}$ \\
\hline $\mathrm{Bt}$ & $0,35-0,73$ & $1,54 \mathrm{Aa}$ & $22 \mathrm{ABa}$ & $21 \mathrm{Aa}$ & $43 \mathrm{Aa}$ \\
\hline \multicolumn{6}{|c|}{ Perfil T30 } \\
\hline Ap & $0,00-0,18$ & $1,46 \mathrm{Aa}$ & $25 \mathrm{Aa}$ & $21 \mathrm{Aa}$ & $46 \mathrm{Aa}$ \\
\hline $\mathrm{AB}$ & $0,18-0,30$ & $1,67 \mathrm{Aa}$ & $27 \mathrm{Aa}$ & $10 \mathrm{Aa}$ & 39Aа \\
\hline $\mathrm{Bt}_{1}$ & $0,30-0,59$ & $1,53 \mathrm{Aa}$ & $29 \mathrm{Aa}$ & $15 \mathrm{Aa}$ & $44 \mathrm{Aa}$ \\
\hline
\end{tabular}

\footnotetext{
(1)Médias seguidas das mesmas letras, maiúsculas nas linhas e minúsculas nas colunas, não diferem entre si a 5\% de probabilidade pelo teste de Tukey.
} 
A porosidade total não foi alterada de forma significativa, nem com o cultivo e nem com a profundidade nos solos estudados (Tabela 3). Nos perfis cultivados, entretanto, os valores da porosidade total foram inferiores aos valores do perfil $\mathrm{Tn}$ nos horizontes $\mathrm{Ap}$ e $\mathrm{AB}$, mais afetados pelo cultivo.

O uso contínuo do solo com o cultivo de cana-deaçúcar causou um expressivo aumento da microporosidade. No horizonte superficial, os efeitos foram imediatos, em conseqüência do desmatamento (T2) e preparo do solo, tendendo a se estabilizar com o maior tempo de cultivo (T30). Nos horizontes subsuperficiais, os efeitos foram mais lentos e o aumento da microporosidade se deve à intensa utilização de máquinas comprimindo os poros, e ainda, ao entupimento dos mesmos por argila iluvial, conforme observado por Oliveira et al. (1983), na região de Lavras e por Silva \& Ribeiro (1997), em Latossolos Amarelos coesos dos tabuleiros de Alagoas.

A capacidade de campo (CC) e o ponto de murcha permanente (PMP) aumentaram do horizonte $\mathrm{A}$ e $\mathrm{AB}$ para o Bt, nas três situações, acompanhando o aumento do conteúdo de argila, responsável por um maior poder de retenção de umidade, o que era esperado em um Argissolo e não se relaciona com o efeito de cultivo (Tabela 4). Observou-se que, logo após a retirada da mata e a rápida decomposição da matéria orgânica, houve uma redução na $\mathrm{CC}$ dos horizontes superficiais do perfil $\mathrm{T} 2$, em relação ao Tn, aumentando, posteriormente, com o do tempo de cultivo (T30). Este resultado pode ser explicado pela redução da macroporosidade, aumento da microporosidade e pelo maior teor de matéria orgânica em T30 (Tabelas 2 e 3). Como o PMP não sofreu modificações significativas com o aumento da microporosidade, por refletir um teor de umidade retido a tensões bem mais altas que a CC, observou-se um aumento da disponibilidade de água com o aumento do tempo de cultivo.

Se por um lado a utilização agrícola promoveu um aumento na disponibilidade da água, em função das alterações nas propriedades físicas, como estrutura e microporosidade, por outro lado, estas alterações resultaram em restrições ao crescimento do sistema radicular, limitando o volume de solo explorado pelas raízes e, conseqüentemente, a absorção de água pelas plantas. A redução do crescimento das raízes foi observada pela descrição morfológica, no horizonte $\mathrm{AB}$ dos perfis $\mathrm{T} 2 \mathrm{e}$ T30 (Tabela 1).
Os resultados da condutividade hidráulica saturada revelaram que houve uma redução significativa do fluxo de água no solo da área de mata (Tn) para as áreas com cultivo de cana-de-açúcar (T2 e T30), que não mostraram diferenças significativas entre si. Estes resultados são consequiência da redução da macroporosidade e aumento da microporosidade nos três primeiros horizontes do perfil, causados pela utilização agrícola (Tabela 3). Resultados semelhantes foram constatados por Silva (1996) e Paiva (1997).

Houve um decréscimo nos valores da porcentagem de agregados $>1,00 \mathrm{~mm}$ (macroagregados) no perfil T2, em relação ao perfil sem cultivo, e uma posterior recuperação no perfil com 30 anos (Tabela 5). Esta redução pode ser explicada pelo recente desmatamento e operações de cultivo. No perfil com tempo de cultivo mais prolongado, ao contrário do esperado, observou-se um

Tabela 4. Capacidade de campo, ponto de murcha permanente, água disponível, disponibilidade total de água no solo e condutividade hidráulica saturada em razão do tempo de cultivo e profundidade do solo ${ }^{(1)}$.

\begin{tabular}{|c|c|c|c|}
\hline \multirow{2}{*}{$\begin{array}{l}\text { Tempo de } \\
\text { cultivo }^{(2)}\end{array}$} & \multicolumn{3}{|c|}{ Horizonte } \\
\hline & A & $\mathrm{AB}$ & $\mathrm{Bt}$ \\
\hline & \multicolumn{3}{|c|}{ Capacidade de campo ${ }^{(3)}\left(\mathrm{g} \mathrm{kg}^{-1}\right)$} \\
\hline $\mathrm{Tn}$ & $96,1 \mathrm{ABb}$ & $86,4 \mathrm{ABb}$ & $146,0 \mathrm{ABa}$ \\
\hline $\mathrm{T} 2$ & $84,0 \mathrm{Bb}$ & $92,4 \mathrm{Bb}$ & $147,3 \mathrm{Ba}$ \\
\hline \multirow[t]{2}{*}{$\mathrm{T} 30$} & $104,6 \mathrm{Ab}$ & $100,1 \mathrm{Ab}$ & $164,5 \mathrm{Aa}$ \\
\hline & \multicolumn{3}{|c|}{ Ponto de murcha permanente $\left(\mathrm{g} \mathrm{kg}^{-1}\right)$} \\
\hline $\operatorname{Tn}$ & $49,8 \mathrm{Ab}$ & $48,2 \mathrm{Ab}$ & $107,2 \mathrm{Aa}$ \\
\hline $\mathrm{T} 2$ & $35,1 \mathrm{Ab}$ & $45,1 \mathrm{Ab}$ & $105,6 \mathrm{Aa}$ \\
\hline \multirow[t]{2}{*}{$\mathrm{T} 30$} & $44,2 \mathrm{Ab}$ & $50,3 \mathrm{Ab}$ & $112,4 \mathrm{Aa}$ \\
\hline & \multicolumn{3}{|c|}{ Água disponível $\left(\mathrm{g} \mathrm{kg}^{-1}\right)$} \\
\hline $\operatorname{Tn}$ & $46,3 \mathrm{Ba}$ & $38,2 \mathrm{Ba}$ & $38,8 \mathrm{Ba}$ \\
\hline $\mathrm{T} 2$ & $48,9 \mathrm{ABa}$ & $47,3 \mathrm{ABa}$ & $41,7 \mathrm{ABa}$ \\
\hline \multirow[t]{2}{*}{$\mathrm{T} 30$} & $60,4 \mathrm{Aa}$ & $49,8 \mathrm{Aa}$ & $52,1 \mathrm{Aa}$ \\
\hline & \multicolumn{3}{|c|}{ Disponibilidade total de água do solo $\left(\mathrm{mm} \mathrm{dm}^{-3}\right)$} \\
\hline $\operatorname{Tn}$ & $0,55 \mathrm{Ba}$ & $0,54 \mathrm{Ba}$ & $0,61 \mathrm{Ba}$ \\
\hline $\mathrm{T} 2$ & $0,74 \mathrm{Aa}$ & $0,75 \mathrm{Aa}$ & $0,64 \mathrm{Aa}$ \\
\hline \multirow[t]{2}{*}{ T30 } & $0,88 \mathrm{Aa}$ & $0,83 \mathrm{Aa}$ & $0,80 \mathrm{Aa}$ \\
\hline & \multicolumn{3}{|c|}{ Condutividade hidráulica saturada $\left(\mathrm{cm} \mathrm{h}^{-1}\right)$} \\
\hline $\mathrm{Tn}$ & $55,8 \mathrm{Aa}$ & $22,4 \mathrm{Ab}$ & $21,9 \mathrm{Ab}$ \\
\hline $\mathrm{T} 2$ & $23,9 \mathrm{Ba}$ & $19,7 \mathrm{Bb}$ & $11,6 \mathrm{Bb}$ \\
\hline T30 & $17,0 \mathrm{Ba}$ & $11,6 \mathrm{Bb}$ & $6,0 \mathrm{Bb}$ \\
\hline
\end{tabular}

${ }^{(1)}$ Médias seguidas das mesmas letras, maiúsculas nas colunas e minúsculas nas linhas, não diferem entre si a $5 \%$ de probabilidade pelo teste de Tukey. ${ }^{(2)} \mathrm{Tn}$ : mata nativa; T2: dois anos; T30: trinta anos. ${ }^{(3)}$ Para análise mais precisa da água disponível usou-se nos horizontes $\mathrm{A}$ e $\mathrm{AB}$ pressão de 0,01 MPa e no horizonte Bt a pressão de 0,033 Mpa. 
Tabela 5. Porcentagem de agregados no horizonte A ou Ap retidos em peneiras de 2,0,1,0, 0,5 e 0,25 mm, agregação e estabilidade de agregados na fração $>0,5 \mathrm{~mm}$ em razão do tempo (T) de cultivo com cana-de-açúcar $\left(2\right.$ e 30 anos ${ }^{(1)}$.

\begin{tabular}{|c|c|c|c|c|c|c|}
\hline \multirow{2}{*}{$\begin{array}{l}\text { Tempo de } \\
\text { cultivo }\end{array}$} & \multicolumn{4}{|c|}{ Diâmetro das peneiras (mm) } & \multirow[t]{2}{*}{ Agregação } & \multirow{2}{*}{$\begin{array}{l}\text { Estabilidade } \\
\text { de agregados }\end{array}$} \\
\hline & 2 & 1 & 0,5 & 0,25 & & \\
\hline & \multicolumn{6}{|c|}{ 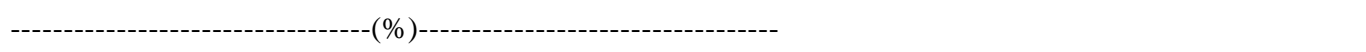 } \\
\hline $\operatorname{Tn}^{(2)}$ & 14,31 & 10,70 & 32,32 & 27,04 & $40 \mathrm{~A}$ & $69 \mathrm{~A}$ \\
\hline $\mathrm{T} 2$ & 6,94 & 10,85 & 43,70 & 22,92 & $40 \mathrm{~A}$ & $65 \mathrm{~A}$ \\
\hline $\mathrm{T} 30$ & 13,77 & 14,24 & 40,07 & 17,80 & $45 \mathrm{~A}$ & $67 \mathrm{~A}$ \\
\hline
\end{tabular}

${ }^{(1)}$ Médias seguidas de letras iguais não diferem entre si pelo teste de Tukey a 5\% de probabilidade. ${ }^{(2)} \mathrm{Tn}$ : mata nativa.

aumento na porcentagem destes agregados. Os horizontes superficiais dos perfis Tn e T2 apresentaram valores semelhantes de agregação em torno de $40 \%$, e menores que o T30. No entanto, o Tn teve maiores valores para o índice de estabilidade de agregados, seguido do T30, apesar de, tanto para agregação quanto para estabilidade de agregados, não terem ocorrido diferenças significativas em relação ao cultivo (Tabela 5).

\section{Conclusões}

1. Os horizontes superficiais dos Argissolos Amarelos distróficos abrúpticos fragipânicos, em virtude dos baixos teores de argila, apresentam modificações morfológicas pouco expressivas, decorrentes do cultivo da cana-de-açúcar.

2. Ocorrem modificações na topografia da transição do horizonte $\mathrm{Ap}$ para o $\mathrm{AB}$, e do $\mathrm{AB}$ para o $\mathrm{Bt}$, e na estrutura dos horizontes $\mathrm{Ap}$ e $\mathrm{AB}$ dos perfis decorrentes do cultivo da cana-de-açúcar.

3. O cultivo do solo provoca aumento na microporosidade e na água disponível, e redução na condutividade hidráulica saturada dos horizontes Ap e $\mathrm{AB}$ dos perfis.

4. O cultivo da cana-de-açúcar causa o desenvolvimento de compactação nos horizontes Ap e AB; constata-se a existência de adensamento (caráter coeso) no horizonte $\mathrm{Bt}$.

\section{Referências}

CAMARGO, O.A. Efeitos da compactação em características do solo. In: Compactação do solo e desenvolvimento de plantas. Campinas: Fundação Cargill, 1983. 44p.

CAMARGO, O.A.; ALLEONI, L.R.F. Compactação do solo e desenvolvimento das plantas. Piracicaba: Esalq, 1997. 132p.

CAVENAGE, A.; MORAES, M.L.T.; ALVES, M.C.; CARVALHO, M.A.C.; FREITAS, M.L.M.; BUZETTI, S. Alterações nas propriedades físicas de um Latossolo Vermelho-Escuro sob diferentes culturas. Revista Brasileira de Ciência do Solo, v.23, p.997-1003, 1999.

CINTRA, F.L.D. Caracterização do impedimento mecânico em Latossolo do Rio de Grande do Sul. 1980. 100p. Dissertação (Mestrado) - Universidade Federal do Rio Grande do Sul, Porto Alegre.

CORREIA, J.C. Efeito de métodos de cultivo em algumas propriedades físicas de um Latossolo Amarelo muito argiloso do Estado do Amazonas. Pesquisa Agropecuária Brasileira, v.20, p.1317-1322, 985.

DA ROS, C.O.; SECCO, D.; FIORIN, J.E.; PETRERE, C.; CADORE, M.A.; PASA, L. Manejo do solo a partir de campo nativo: efeito sobre a forma e estabilidade da estrutura ao final de cinco anos. Revista Brasileira de Ciência do Solo, v.21, p.241$247,1997$.

EMBRAPA. Centro de Pesquisas Pedológicas (Rio de Janeiro, RJ). Levantamento exploratório-reconhecimento de solos do Estado de Alagoas. Recife, 1975. 532p. (Embrapa-CPP. Boletim Técnico, 35; SUDENE. Série Recursos de Solos, 5).

EMBRAPA. Centro Nacional de Pesquisa de solos (Rio de Janeiro, RJ). Manual de métodos de análises de Solo. 2.ed. rev. atual. Rio de Janeiro, 1997. 212p.

EMBRAPA. Serviço Nacional de Levantamento e Conservação de Solos (Rio de Janeiro, RJ). Manual de métodos de análises de solos. Rio de Janeiro, 1979. 247p.

GROHMANN, F. Análises de agregados de solos. Bragantia, v.19, p.201-213, 1960.

KLUTE, A.; DIRKSEN, C. Hydraulic conductivity and diffusivity laboratory methods. In: KLUTE, A. (Ed.). Methods of soil analysis: physical and mineralogical methods. Madison: American Society of Agronomy, 1986. part 1. p.687-732.

LEMOS, R.C.; SANTOS, R.D. Manual de descrição e coleta de solo no campo. 2.ed. Campinas: Sociedade Brasileira de Ciência do Solo, 1996. 83p.

LIMA, J.P.R. O setor sucro-alcooleiro do Nordeste: evolução recente e a reestruturação possível. In: WORKSHOP SOBRE AVALIAÇÃO E MANEJO DOS RECURSOS NATURAIS EM ÁREA DE EXPLORAÇÃO DA CANA-DE-AÇÚCAR, 1997, Aracaju. Palestras. Aracaju: Embrapa-CPATC, 1997. p.9-32. 
MAHBOUBI, A.A.; LAL, R.; FAUSSEY, N.R. Twenty-eight year of tillage effects on two soils in Ohio. Soil Science Society of America Journal, v.57, p.506-512, 1993.

OLIVEIRA, M.; CURI, N.; FREIRE, J.C. Relações massas/volume em Podzólico Vermelho-Amarelo textura média/argilosa da região de Lavras (MG) sob pastagens e cultivo anual. Ciência e Prática, v.7, p.66-74, 1983.

PAIVA, A. de Q. Dinâmica da água em uma topossequiência de solos de tabuleiro do Estado da Bahia e sua implicação no crescimento da laranjeira. 1997. 74p. Dissertação (Mestrado) Universidade Federal de Viçosa, Viçosa.

RICHARDS, L.A. (Ed.). Diagnosis and improvement of saline and alkaline soils. Washington: United States Department of Agriculture, 1969. 160p. (Agriculture Handbook, 60).

SAS INSTITUTE (Cary, Estados Unidos). SAS/STAT user's guide: version 8. Cary, 1999. 3884p.

SILVA, A.J.N. da. Caracterização de Latossolos amarelos sob cultivo contínuo de cana-de-açúcar no Estado de Alagoas. 1996. 133p. Dissertação (Mestrado) - Universidade Federal Rural de Pernambuco, Recife.

SILVA, A.J.N. da; RIBEIRO, M.R. Caracterização de Latossolo amarelo sob cultivo contínuo de cana-de-açúcar no Estado de Alagoas: atributos morfológicos e físicos. Revista Brasileira de Ciência do Solo, v.21, p.677-684, 1997.
SILVA, I. de F. da. Efeitos de sistemas de manejo e tempo de cultivo sobre propriedades físicas de Latossolo. 1980. 105p. Dissertação (Mestrado) - Universidade Federal do Rio Grande do Sul, Porto Alegre.

SILVA, J.A A. da; SILVA, I.P. da. Estatística experimental aplicada à Ciência Florestal. Recife: Imprensa Universitária da UFRPE, 1995. 296p.

SILVA, M.S.L.; RIBEIRO, M.R. Influência do cultivo da cana-deaçúcar em propriedades morfológicas e físicas de solos argilosos de tabuleiro no Estado de Alagoas. Revista Brasileira de Ciência do Solo, v.16, p.397-402, 1992.

URQUIAGA, S.; RESENDE, A.S. de; ALVES, B.J.R.; BODDEY, R.M.; DÖBEREINER, J. Fixação biológica de nitrogênio na cultura da cana-de-açúcar: perspectivas. In: REUNIÃO TÉCNICA SOBRE SOLOS COESOS DOS TABULEIROS COSTEIROS, 1996, Cruz das Almas. Anais. Aracaju: Embrapa-CPATC, 1996. p.39-48.

USINA CORURIPE. (Coruripe, AL). Levantamento detalhado de solos da Usina Coruripe. Coruripe, 1997. 47p.

VOMOCIL, J.A. Porosity. In: BLACK, C.A. (Ed.). Methods of soil analysis: physical and mineralogical properties, including statistics of measurement and sampling. Madison: America Society Agronomy, 1965. pt.1, p.299-314.

WU, L.; SWAN, J.B.; ALLMARAS, R.R.; LOGSDON, S.D. Tillage and traffic influence on water and solute transport in corn-soybean systems. Soil Science Society of America Journal, v.59, p.185$191,1995$.

Recebido em 11 de junho de 2003 e aprovado em 6 de novembro de 2003 